\title{
MULTIPLE-TRAJECTORY-PREDICTION (MTP) ALGORITHM FOR UAS'S SENSE AND AVOID (SAA) OPERATION
}

\author{
Yan Zhang and Gerard Fairley \\ U.S. DOT Volpe National Transportation Systems Center, Cambridge, Massachusetts
}

\begin{abstract}
This paper investigates the Sense And Avoid (SAA) system for Unmanned Aircraft Systems (UASs) with a Multiple-Trajectory-Prediction (MTP) algorithm that can potentially operate in all categories defined by the U.S. Federal Aviation Administration (FAA) [1]. The MTP algorithm selects avoidance maneuvers for the aircraft to obtain the maximum separation by running simulations in an on-board SAA computer. The algorithm searches for the best maneuver parameters using the data from advanced surveillance systems such as the Automatic Dependent Surveillance-Broadcast (ADS-B).

The proposed SAA system with MTP algorithm could be used as the Common Algorithm (CA) for UAS SAA operations with lower cost and higher accuracy compared to the existing systems such as TCAS.
\end{abstract}

\section{Introduction}

In the past few decades a rapid increase of UAS of the U.S. military and commercial applications has occurred. Even without the introduction of UASs the national airspace (NAS) is becoming crowded. It is desired to merge the UASs into the NAS without sacrificing safety. The U.S. Federal Aviation Administration (FAA) recently released the Concept of Operations (CONOPS) for Integration of UASs into the NAS to address, in particular, this issue [1]. The Sense and Avoid (SAA) system was listed as one of the major requirements for UASs to be integrated into the NAS.

\section{Overview of Traffic Collision Avoidance System (TCAS)}

The concept of Traffic Collision Avoidance System (TCAS) [2], was considered as a basic requirement for the development of UAS's SAA systems. TCAS is a mid-air collision avoidance system (CAS) that provides vertical avoidance maneuvers and enhanced situational awareness.
However, the current TCAS model only provides instructions to climb or descend. When TCAS interrogates nearby aircraft, communication consists of secondary surveillance radar (SSR) transponder signals. TCAS operates independently of aircraft navigation, flight management systems, and groundbased equipment. Although TCAS has been developed for decades, we believe it must be modified and improved to fulfill the requirements of the UAS integration in the NAS, such as cost reduction, adding lateral maneuver to achieve maximum separation and simplifying the avoidance algorithm to increase the accessibility and robustness.

There are two main versions of TCAS: TCAS I and TCAS II. TCAS I provides Traffic Advisories (TA) and proximity warnings. TCAS I is required for use in the United States by turbine-powered passenger carrying aircraft. TCAS I is also used by some general aviation fixed and rotary wing aircraft. TCAS I is the first generation of collision avoidance technology. It is cheaper but less capable than the modern TCAS II system. TCAS I systems monitor the traffic around a plane (to about 30 miles) and can provide the approximate bearing and altitude of the other aircraft. It can also generate collision warnings in the form of a TA. The TA warns the pilot, "traffic, traffic" that another aircraft is nearby, but does not compute a solution. However, the TA information does aid in the "see and avoid" action. The pilot must decide a course of action, usually with the assistance of Air Traffic Control [3]. TCAS II provides all the benefits of TCAS I, but also provides the pilot vocal instructions to avoid accidents, known as Resolution Advisory (RA). The RA provides vertical escape paths that either increase or maintain vertical separation between aircraft. TCAS II provides both TAs and RAs. TCAS II is used in most commercial aircraft.

TCAS III was planned as a horizontal and vertical avoidance system but was determined currently unfeasible due to lack of horizontal position accuracy. Currently, research is being conducted to develop a future collision avoidance system (under

U.S. Government work not protected by U.S. copyright 
the working name of Aircraft Collision Avoidance System (ACAS X). If developed and certified, ACAS $\mathrm{X}$ will not be commercially available before middle 2020s. It is unclear at this stage whether ACAS X would provide any horizontal resolutions. Our proposed SAA model would be a competitor to the ACAS X model.

TCAS aircraft avoidance maneuvers are restricted to changes in altitude and modification of climb/descend rates. After identifying potential collisions, an avoidance maneuver algorithm between the two (or up to 30) aircraft automatically negotiates conflicting aircraft. Multi-threat logic utilizes increased rate RAs and reversal RAs to best resolve multi-threat conditions.

TCAS uses the same radar transponders as those used by aircraft to communicate with Air Traffic Control (ATC) ground-based radars. The level of protection provided by TCAS equipment depends on the type of transponder aircrafts carry. The level of protection is given in Table 1.

Table 1. TCAS Protection Levels [4]

\begin{tabular}{|c|c|c|c|}
\hline & \multicolumn{2}{|c|}{ TCAS Aircraft } \\
\hline & & TCAS I & TCAS II \\
\hline \multirow{4}{*}{ 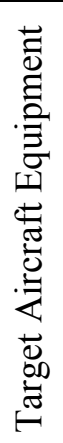 } & $\begin{array}{l}\text { Mode A XPDR } \\
\text { only }\end{array}$ & TA & TA \\
\hline & $\begin{array}{c}\text { Mode C or } \\
\text { Mode S XPDR }\end{array}$ & TA & $\begin{array}{c}\text { TA and } \\
\text { Vertical RA }\end{array}$ \\
\hline & TCAS I & TA & $\begin{array}{c}\text { TA and } \\
\text { Vertical RA }\end{array}$ \\
\hline & TCAS II & TA & $\begin{array}{c}\text { TA and } \\
\text { Coordinated } \\
\text { Vertical RA }\end{array}$ \\
\hline
\end{tabular}

TCAS provides no protection from aircraft not equipped with a transponder. TCAS works autonomously of aircraft navigation equipment and the ground ATC systems.

TCAS interrogates transponders of all aircraft 'nearby'. Based on the replies, it tracks the slant range, altitude, and bearing of each aircraft. TCAS can issue two types of alerts: TA and RA. The TA traffic display gives the position of nearby traffic. The TA helps the pilot visually search for the detected traffic and helps in preparation for an RA if necessary. If the TA is issued and the traffic is visually acquired, pilots are instructed to maintain visual separation from the traffic. The RA display provides the pilot with information on the vertical speed. RA's recommended maneuvers will either increase or maintain the existing vertical separation from an intruder aircraft. The autopilot must be turned off and the RA avoidance procedure flown manually (flight director and ATC advisories must be ignored).

The tau time, calculated by the distance between the own aircraft and the intruder divided by the closure rate, plays an important role for TCAS and other SAA systems. When aircraft are on a collision course tau is the actual time to Closest Point of Approach (CPA) and tau will be zero at collision. Tau is only an approximation of time to CPA if the aircraft will only pass near each other. If tau is only an approximation then tau will decrease to a minimum value before actual CPA and increase afterward.

\section{Overview of Sense and Avoid (SAA) System}

The traditional SAA for UASs includes TCAS, electro-optical (EO) sensors that produce images that mimic human sight, and Ground Based Sense And Avoid (GBSAA) using primary radar. The SAA requires a UAS to remain well clear from and avoid collisions with other airborne traffic [5]. The SAA is also required to compensate for the lack of an onboard pilot, and to define an operational concept that will enable the SAA-equipped aircraft to smoothly integrate into an air traffic services environment [6]. This will be accomplished by a computer model, Common Algorithm (CA), similar in scope as TCAS but utilizing a different algorithm. The SAA should be equal to or better than the See And Avoid capability of a manned aircraft.

The ADS-B-Out transmitter is effectively a Mode S transponder using 1090ES. In ten years, ADS-B-Out and ADS-B-In are scheduled to be used instead of Mode $\mathrm{S}$ when operating TCAS. The common algorithm (CA) is designed for this future use and thus incorporates the ADS-B functionality. Since CA can replace TCAS then CA can be used to help fulfill SAA.

Each UAS has a control link and a communication link, enabling the Pilot in Command (PIC) to control the vehicle by radio or satellite and 
communicate with ATC. In addition, the PIC may communicate with the ATC via a ground relay. The voice communication link between the UAS PIC and ATC can lack the reliability of the link between manned aircraft pilots and ATC since there is in general an additional link for the PIC. The link also experiences additional latencies and degradations due to the atmosphere. The Unmanned Aircraft (UA) may be beyond radio coverage from the PIC. A satellite relay links the PIC to the UA to deliver voice communications between the UA and the ATC. This adds a communications delay. These conditions introduce some amount of control and communication latency. The combined delays in receipt of and response to ATC instructions may adversely affect aircraft separation and control and hence the safety of other elements of the NAS and ground infrastructure. Since link loss procedures are generally not part of all ATC automation systems ATC may not be immediately aware that a lost link has occurred. UAS response to a link loss may result in the UA changing course or altitude without clearance.

UAS can be integrated with technologies and procedures such as Performance-based Navigation $(\mathrm{PBN})$, digital voice switching, and data communications (DATACOM). When combined with Trajectory-Based Operations (TBO) and decision support tools, these can provide the foundation for managing UASs. UAS responsibilities for safe separation include SAA. SAA represents the capability to provide safe separation from other traffic. SSA has two functional components:

1. Self-separation - functions to perform separation analogous to the visually-based requirement for manned aircraft to remain well separated from other aircraft.

2. Collision avoidance - a few large UAS are equipped with TCAS. For the other UASs this function also serves to provide maneuver advisories similar to TCAS for avoiding mid-air collision with another aircraft. The CA can potentially bring TCAS-like protection to even much smaller UAs.

These two functions of SAA must give a capability analogous to the visually-based requirements. For these functions the CA system can replace the TCAS system and act to achieve self- separation. The technologies these are based on must be at least equivalent with manned capabilities. Data from airborne sensors, ADS-B-Out messages, ground-based radar or other inputs, could be used to achieve SAA capability. Large enough UAS will be equipped with ADS-B-Out and transponder.

Autonomous operations (system design that prevents any person from affecting the normal operations of the aircraft) are not permitted. The PIC has full control, to assume control at all times during normal UAS operations.

SAA self-separation is different from the manned aircraft requirement to "remain well clear" in that it calculates using a set of quantitative values to determine if an avoidance maneuver is necessary. These maneuvers can be carried out using the CA algorithm. A UA is authorized to use its selfseparation capability for encounters between itself and an aircraft on Visual Flight Rules (VFR) (which is not receiving ATC services). In this case, selfseparation is the responsibility of the pilots of both aircraft.

Regardless of whether ATC separation services are provided, the PIC achieves collision avoidance using SAA (with systems such as TCAS or CA). The UAS takes action to prevent another aircraft from penetrating the UAS collision volume. The UAS PIC ensures the collision avoidance function is always active. Otherwise, ATC is notified.

ADS-B-Out transmits and ADS-B-In receives aircraft position and other state information. ADS-B-Out improves the probability that both ATC and other equipped aircraft can detect the UA. It also gives other aircraft equipped with ADS-B-In a better chance to remain well clear. This is consistent with the $\mathrm{CA}$ algorithm that it requires ADS-B data.

\section{Multiple-Trajectory-Prediction (MTP) Based Sense and Avoid (SAA)}

This paper introduces a new algorithm for making decisions of how to maneuver the UAS and/or the "intruder" aircraft to obtain the maximum separation. This algorithm is based on the so-called Multiple-Trajectory-Prediction (MTP) that searches for the best flight directions from several tries based on the input of aircraft state vectors observed by the advanced surveillance system such as the ADS-B. This process can be repeated after a TA is issued to 
achieve higher level of confidence and/or make corrections when flight or environment conditions are changed after the initial avoidance actions are taken. The moment when the MTP-based SAA is executed is equivalent to the moment when RA for a TCAS is issued.

\section{System Design}

The information flow chart shown in Figure 1 illustrates the simulation procedure of the MTP-based SAA system. In the simulation, the aircraft flight dynamics, both for the own aircraft and intruder, is the kinematic model (Appendix A) which calculates time-stamped flight state vectors, including latitude/longitude/altitude locations, indicated and true airspeeds (Appendix B), magnetic and true heading angles, and angles of bank.

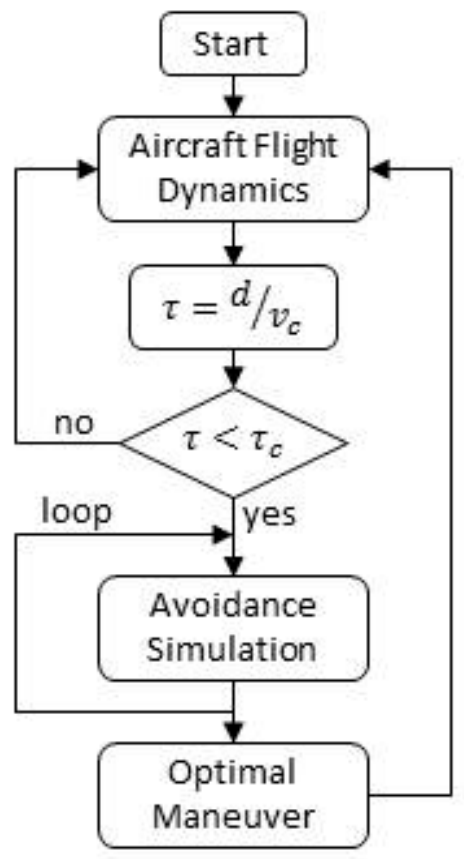

\section{Figure 1. Information Flow Chart for Multiple- Trajectory Prediction Based SAA Simulation}

The predicted time of collision, tau ( $\tau$ ), is calculated using the line-of-sight distance and the closure rate $\left(v_{c}\right)$ between the aircraft. The tau $(\tau)$ is compared with the critical value $\left(\tau_{c}\right)$. If $\tau>\tau_{c}$, indicating that the two aircraft are still far away, the simulation continues and the aircraft flight is considered as normal. If $\tau \leq \tau_{c}$, then the avoidance simulation starts with loops to search for maneuver directions (see Figure 2) for maximum separations.

The aircraft then perform the avoidance actions based on the flight directions defined by the selected maneuver code.

\section{Maneuver Code}

Figure 2 illustrates the possible turning and climbing/descending directions for aircraft to avoid collisions. In the simulation and/or the proposed real system, a maneuver code is specified by capital letters where the first two defines the maneuver directions for the first aircraft (own aircraft), and the third and fourth letters are used to specify the maneuvers for the second aircraft (intruder). The maneuvers for more aircraft can be specified by letters following the previous ones.
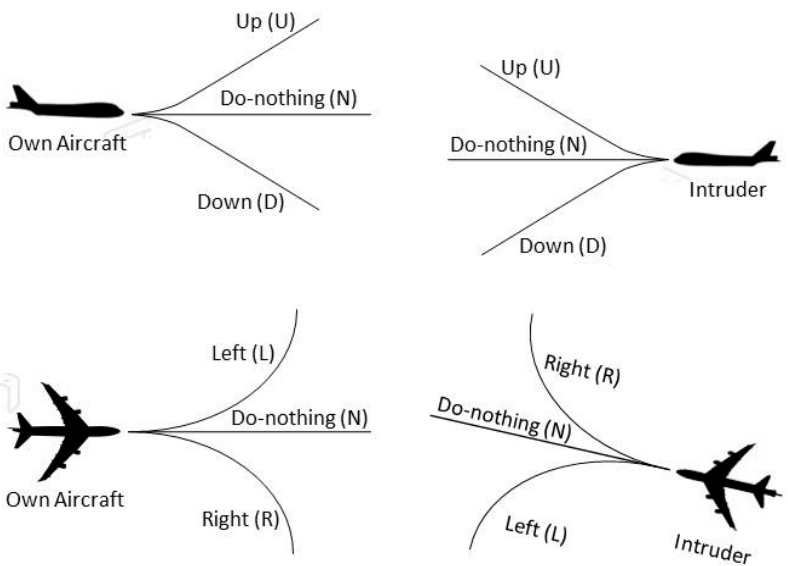

Figure 2. Assumed Aircraft Maneuver Directions for MTP-Based SAA Operation

For example, as illustrated in Figure 3, the LDLU code states that the own aircraft turns to Left and descends (Down) and the intruder turns to Left and climbs (Up) to make the separation. There are three possible choices for the letters specifying the horizontal turning directions (do-Nothing, Left, or Right) and three letters for the vertical turning directions (do-Nothing, Up, and Down). Therefore for the two-aircraft SAA situation, there are $3 \times 3 \times 3 \times 3=81$ possibilities in total. Practically there are 80 possible maneuvers since both aircraft doing nothing will excluded from the list. In addition, the actual list of directions will be much shorter than 80 due to the restriction of the maneuvers. For 
example, for TCAS-like SAA, only the vertical maneuvers are allowed; therefore the letters that specify the horizontal maneuvers for both aircraft must be "N". The code can be extended for morethan-two aircraft SAA situation.

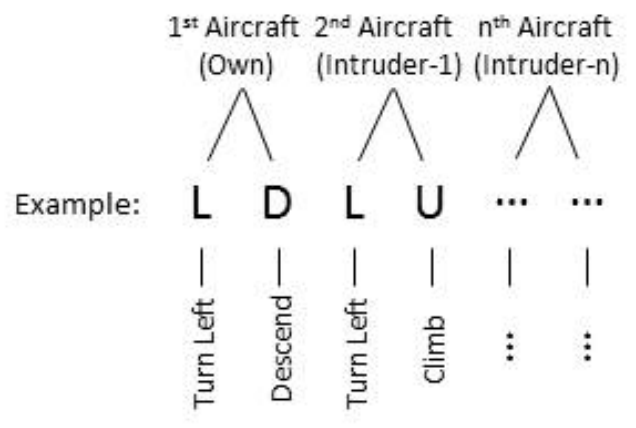

Figure 3. Example Maneuver Code LDLU

\section{Computer Simulation}

\section{Simulation Conditions}

The simulation was performed on a Windows machine with an Intel ${ }^{\circledR}$ Core $^{\mathrm{TM}} 2$ Duo CPU at 2.40GHz. The simulation code was written using Scilab version 5.4.0, an open source software for numerical computation. The simulation adopts the WGS-84 elliptical Earth coordinates, and the time sampling resolution is 0.10 second. Other parameters used in the simulations include

The critical tau: $\tau_{c}=30 \mathrm{sec}$

Angle of bank for avoidance maneuver: $10^{\circ}$

Acceleration of climbing: $1.5 \mathrm{~m} / \mathrm{s}^{2}$

Acceleration of descending: $0.5 \mathrm{~m} / \mathrm{s}^{2}$

The cases for the simulation are listed in Table 2. Although there are 80 (excluding Donothing for both aircraft) possible maneuver combinations, the actual number of maneuver codes could be much less for the specific cases. For example, if the two aircraft can only perform vertical separations with TCAS (Case 1), there should only be two possible maneuver codes for this case: NUND and NDNU. Notice that, no-turning and no-vertical maneuvers are included in Cases 2 and 3 since they may provide larger separations.
Table 2. Sample SAA Maneuver Combinations

\begin{tabular}{|c|c|c|c|}
\hline Case & Own AC & Intruder & $\begin{array}{l}\text { Maneuver } \\
\text { Code }\end{array}$ \\
\hline 1 & $\begin{array}{c}\text { Vertical } \\
\text { only }\end{array}$ & $\begin{array}{c}\text { Vertical } \\
\text { only }\end{array}$ & NUND NDNU \\
\hline 2 & $\begin{array}{l}\text { Horizontal } \\
\text { and } \\
\text { Vertical }\end{array}$ & Do nothing & $\begin{array}{l}\text { NUNN NDNN } \\
\text { LUNN LDNN } \\
\text { RUNN RDNN }\end{array}$ \\
\hline 3 & $\begin{array}{c}\text { Horizontal } \\
\text { and } \\
\text { Vertical }\end{array}$ & $\begin{array}{l}\text { Horizontal } \\
\text { and } \\
\text { Vertical }\end{array}$ & $\begin{array}{l}\text { NULD NDLU } \\
\text { NURD NDRU } \\
\text { LUND LDNU } \\
\text { RUND RDNU } \\
\text { LULD LDLU } \\
\text { RURD RDRU }\end{array}$ \\
\hline
\end{tabular}

\section{Aircraft Initial Positions}

Both the own aircraft and the intruder are initially placed randomly on a circle with radius of 8 nautical miles, heading toward the center of the circle. In order to simulate the worst-case scenario when the two aircraft may collide, the difference of the heading angles between the intruding and own aircraft is larger than $45^{\circ}$, and the altitudes and indicated airspeeds are the same.

Before engaging any SAA maneuvers, it is assumed that the two aircraft fly with a constant indicated speed of 180 knots at the same altitude. It is also assumed that there is no random error assigned to the heading angles, so that the two aircraft are expected to collide at the center of the circle if no SAA actions are taken. More sophisticated simulation or a real system with the proposed MTP algorithm implemented should consider the effect of the errors and uncertainties.

\section{Sense and Avoid}

Like a traditional TCAS, it is proposed that the MTP-based SAA system also constantly monitor the predicted time of collision, tau $(\tau)$. The tau is modeled simply as the division of the distance (see Appendix C) and the closure rate between aircraft, where the closure rate is calculated using the differential formula described in Appendix D. The MTP algorithm starts to simulate the flight paths for various possible flight directions when the tau is less than a critical value $\left(\tau_{c}\right)$, and the "best" maneuver 
code is chosen for each case shown in Table 2 to achieve the largest separation.

\section{Simulation Result}

Simulations are performed to demonstrate the concept of the MTP-based SAA. Various cases are considered to show possibly different performances with various avoidance maneuver allowed, including (1) aircraft only allowed to climb or descend, (2) only the own aircraft turns and climbs/descends, and (3) all aircraft are allowed to turn and climb/descend. The MTP algorithm executed in the simulation can be used as on-board software for a real MTP-based SAA system.

\section{Case 1: Vertical Separation for Both Aircraft}

The maneuver codes NUND and NDNU are considered in the simulation for this case. As an example, Figure 4-a shows the top view of the flight paths with the maneuver code NUND from one of 1,000 simulation runs. The colors indicate paths of different mode. The blue path show the aircraft fly normally toward the collision point. The circle and square marks at the end of blue paths indicates "own aircraft" and "intruder". The red dots in the circle and square windows at the left and right-low corners of the figure show the maneuver directions for the corresponding aircraft. For example, the red dot above the horizontal line in the circle window indicates that the own aircraft climbs; while the red dot below the horizontal line in the square window indicates that the intruder descends during avoidance maneuver. The red portions of the flight trajectories indicate that the aircraft are performing climbing/descending maneuver, and the green portions indicate that the closure rate is negative and the aircraft disengage the avoidance maneuver. Figure 4-b shows the side view of aircraft flight paths with blue, red, and green portions indicating normal flight, avoidance maneuver, and disengaging avoidance modes, respectively.

Figure 4-c shows the minimum distances between the aircraft for the first 50 simulation runs. Note that all of the minimum distances are similar (approximately 1,500 ft) due to the assumptions that the indicated airspeed, altitude, and the performance (angle of bank and climb/descent rate) are the same for both aircraft. It is predicted that the distances should vary more for the situation when flight parameters are difference among aircraft.

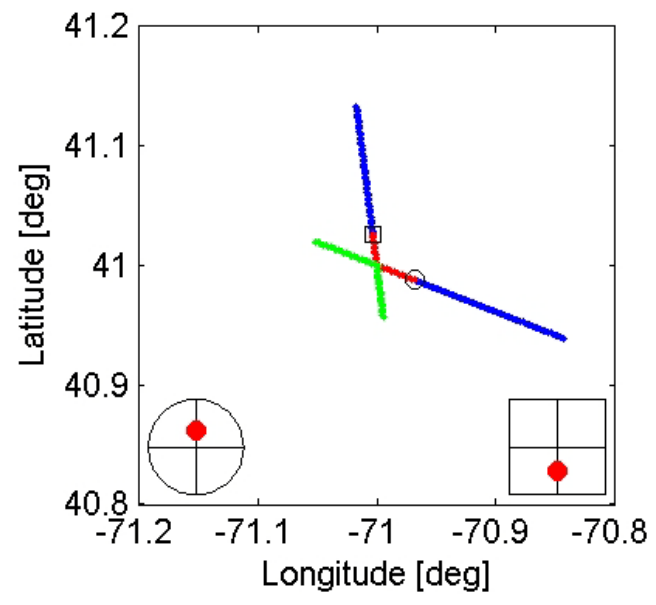

Figure 4-a. Vertical Separation Only by Both Aircraft (NUND)

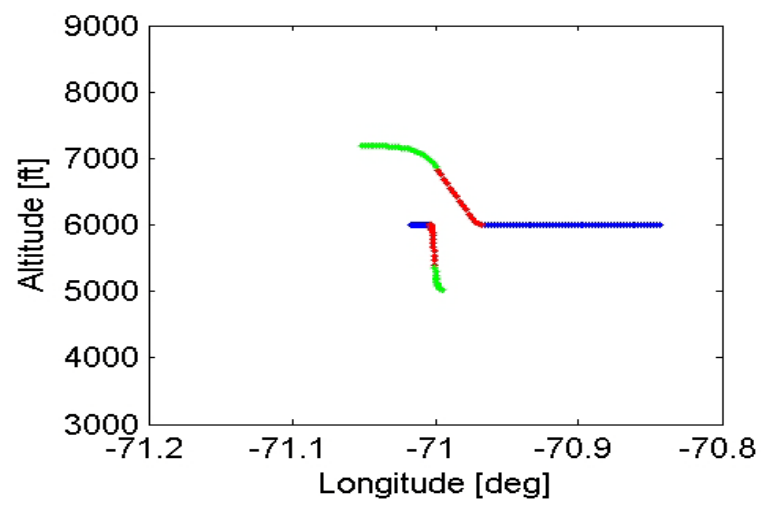

Figure 4-b. Vertical Separation Only by Both Aircraft: Side View

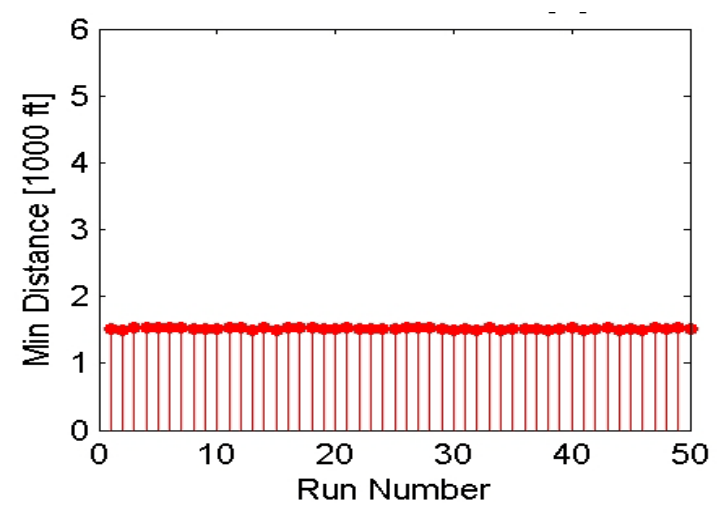

Figure 4-c. Vertical Separation Only by Both Aircraft: Minimum Distance for Each Run 
Case 2: Horizontal and Vertical Separation by Own Aircraft; Intruder Doing Nothing

Six maneuver codes NUNN, NDNN, LUNN, LDNN, RUNN, and RDNN are considered in the simulation for this case. As an example, Figure 5-a and 5-b are the top and side views, respectively, of the flight trajectories with the maneuver code RUNN. The blue path indicates the normal flight mode, red path for avoidance maneuver, and green path for disengaging mode. The circle and square marks at the end of blue paths indicates "own aircraft" and "intruder". For this particular simulation example, the red dot in the circle indicates that the own aircraft climbs and turns right; while the red dot in the square window indicates that the intruder remains the course without doing anything to avoid the collision.

Figure 5-c shows the minimum distances between the aircraft for the first 50 simulation runs for this case. Note that the minimum distances ranges between $2,000 \mathrm{ft}$ and 3,000 ft, which is larger than the separation $(1,500 \mathrm{ft})$ by vertical separation only as shown in Case 1.

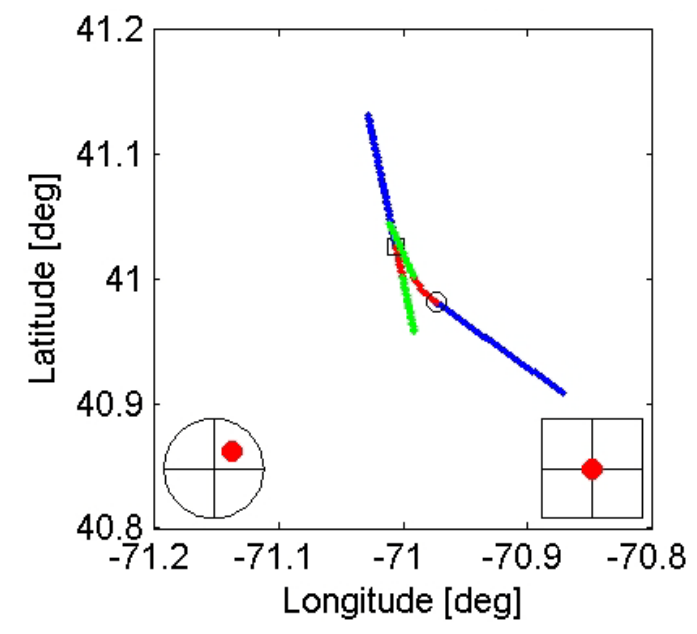

Figure 5-a. Horizontal and Vertical Separation by Own Aircraft Only (RUNN)

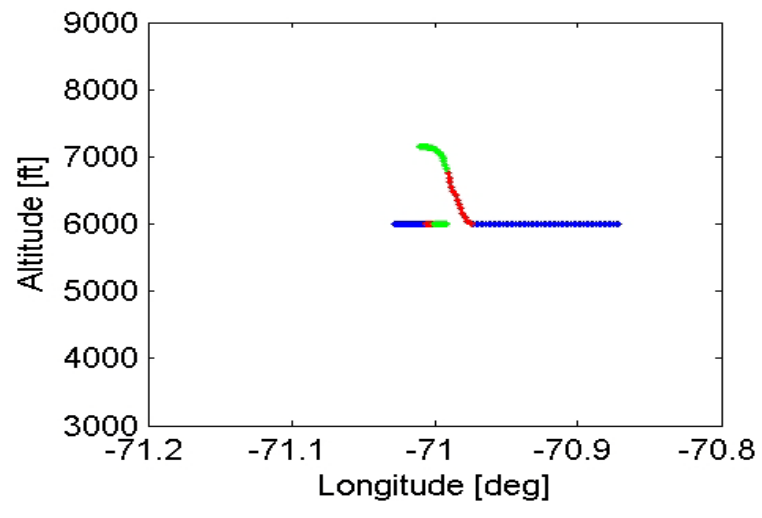

Figure 5-b. Horizontal and Vertical Separation by Own Aircraft Only: Side View

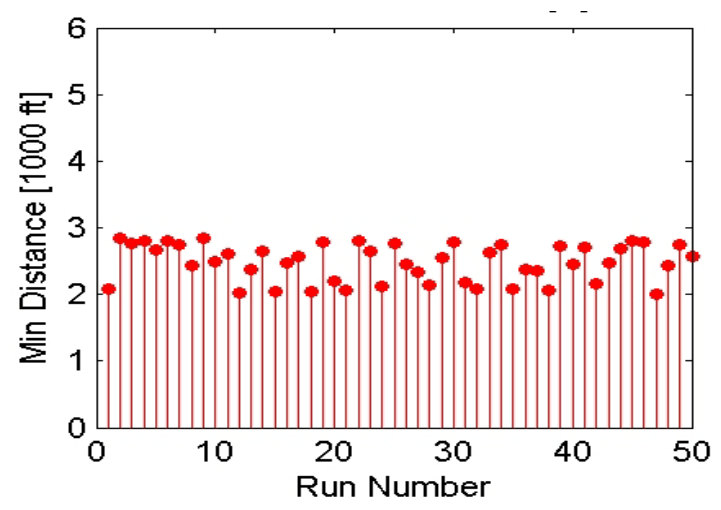

Figure 5-c. Horizontal and Vertical Separation by Own Aircraft Only: Minimum Distance for Each Run

\section{Case 3: Horizontal and Vertical Separation by Both Aircraft}

Twelve maneuver codes are considered for this case as shown in Table 2. As an example, Figure 6-a and 6-b show the top and side views of the flight paths for RURD, respectively. The blue path indicates the normal flight mode, red path for avoidance maneuver, and green path for disengaging mode. The circle and square marks at the end of blue paths indicates "own aircraft" and "intruder". For this particular simulation example, the red dot in the circle indicator shows that the own aircraft turns to right and climbs up; while the intruder turns to right and descends for collision avoidance. 
Figure 6-c shows the minimum distances between the aircraft for the first 50 simulation runs for this case. Note that the distances ranges between $2,600 \mathrm{ft}$ and $5,000 \mathrm{ft}$, larger than the separations by vertical only maneuver performed by both aircraft or the horizontal and vertical maneuver performed by the own aircraft only as shown in Case 1 and Case 2, respectively.

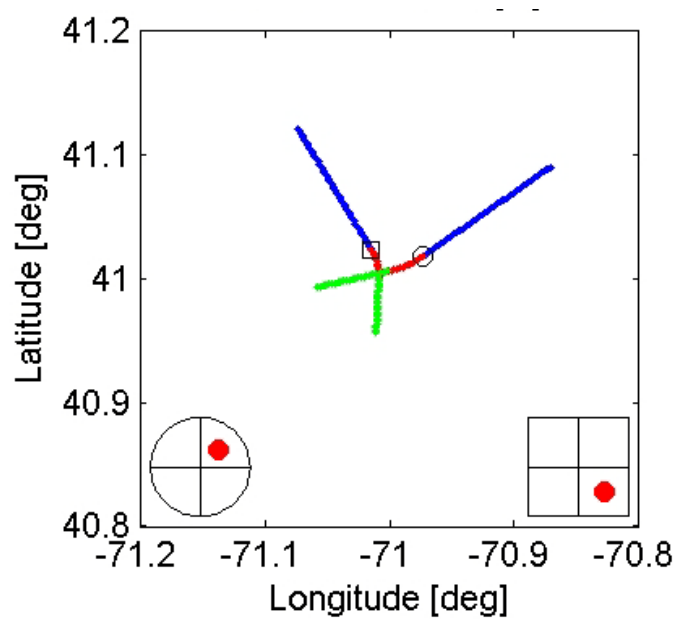

Figure 6-a. Horizontal and Vertical Separation by Both Aircraft (RURD)

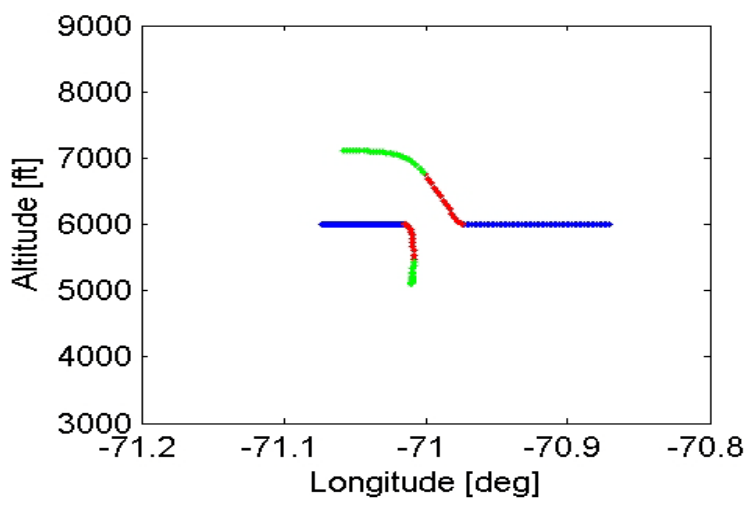

Figure 6-b. Horizontal and Vertical Separation by Both Aircraft: Side View

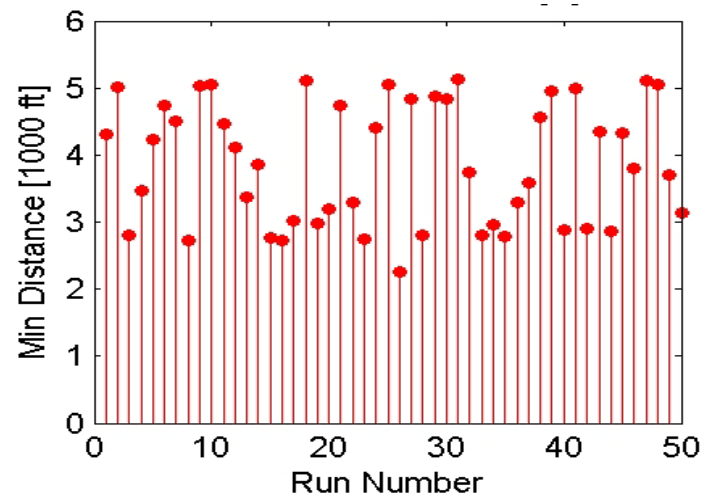

Figure 6-c. Horizontal and Vertical Separation by Both Aircraft: Minimum Distance for Each Run

\section{Selection Rate for Maneuver Code}

The selective rate of the maneuver code for various cases is summarized in Table 3 . For Case 1 when the own aircraft and the intruder only climb or descend, the chances for the own aircraft climbing or the intruder climbing are statistically the same given that the two aircraft are on the same altitude and their climbing or descending rates are the same. For this case, it is important that the two aircraft coordinate through communications to avoid both aircraft climbing or descending at the same time.

For Case 2, when only the own aircraft changes its course and/or altitude (the intruder does nothing), the own aircraft's turning and climbing at the same time (LUNN and RUNN) seems to be more effective to avoid collision than if it only climbs or descends (NUNN and NDNN). Comparing the own aircraft's climbing and descending (LUNN vs. LDNN or RUNN vs. RDNN) the maneuver with climbing is quicker due to the assumption in the simulation that the climbing acceleration is higher than the descending acceleration. Therefore LUNN and RUNN always create larger separations. In addition, the own aircraft's only climbing or descending (NUNN or NDNN) seems unlikely to provide larger separation than other maneuvers.

For Case 3, it is obvious that since both aircraft turn and change altitudes (LULD, LDLU, RURD, and RDRU) they will be more likely to provide larger separation than other maneuvers. If one aircraft does not turn but rather climbs (e.g., NULD, NURD, LDNU, RDNU), it can still provide large separations with lower possibilities. If one aircraft does not turn 
but only descends (e.g., NDLD, NDRD, LDND, RDND), it could not provide the large separation in comparison with other maneuvers because the descending acceleration is lower than the climbing acceleration.

Table 3. Selective Rate of Maneuver Code (per 1,000 runs)

\begin{tabular}{|c|c|c|c|c|}
\hline Case & $\begin{array}{c}\text { Maneuver } \\
\text { Code }\end{array}$ & Count & $\begin{array}{c}\text { Maneuver } \\
\text { Code }\end{array}$ & Count \\
\hline 1 & NUND & 517 & NDNU & 484 \\
\hline \multirow{3}{*}{2} & NUNN & 0 & NDNN & 0 \\
& LUNN & 504 & LDNN & 0 \\
& RUNN & 496 & RDNN & 0 \\
\hline \multirow{6}{*}{3} & NULD & 27 & NDLU & 0 \\
& NURD & 29 & NDRU & 0 \\
& LUND & 0 & LDNU & 34 \\
& RUND & 0 & RDNU & 34 \\
& LULD & 223 & LDLU & 218 \\
& RURD & 213 & RDRU & 222 \\
\hline \multirow{6}{*}{}
\end{tabular}

\section{Real System Integration}

It is assumed that the ADS-B-Out and ADS-BIn will be used instead of Mode $S$ when operating TCAS in ten years. For this future the ADS-B functionality should be available for UASs and to help fulfill SAA. The ADS-B can provide accurate aircraft flight vectors which are sufficient for the MTP-based SAA.

The high-level information flow chart shown in Figure 7 illustrates the structure of the future MTPbased SAA system for UASs. The ADS-B based surveillance system sends the information of aircraft flight state vectors (latitude, longitude, altitude, speed vector, etc.) to the SAA computer to simulate flight trajectories for various maneuver options and select the optimal condition that produces the largest separation. Then the command (maneuver code) is sent to the aircraft for avoidance operations. The advanced technology could make the on-board MTP simulation fast enough to allow frequent update of the simulation and pick up the best maneuver code almost real-time. More sophisticated algorithms could also be adopted to enhance the reliability and accuracy of the decision making by, for example, voting for the decision based on several resultant maneuver codes.
The maneuver code generated by the MTPbased SAA could be used as the CA for the future UAVs since the algorithm is not specific for a particular aircraft, except the number of inputs taken by the SAA computer due to the variability of the surveillance system. Also the maneuver code could be standardized as proposed in this paper to make the SAA be compatible for different aircraft maneuver capabilities.

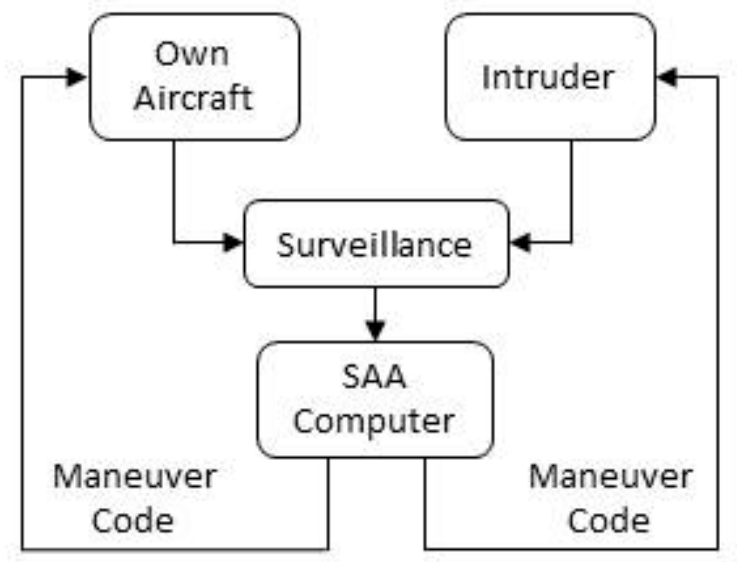

Figure 7. Information Flow Chart for Proposed

\section{Conclusion}

The MTP-based SAA algorithm proposed in this paper could serve as a Common Algorithm (CA) to select the best maneuver parameters for UAS's SAA operations. Based on the same concept, the MTP algorithm can be easily applied to multiple aircraft pairs without significantly altering the logic of operation. Simulation shows that, by horizontal maneuvering, the MTP algorithm could provide larger 3-D separations than the TCAS does where only the vertical separation is performed, even though only one aircraft (own aircraft or intruder) performs the avoidance maneuver.

\section{References}

[1] Federal Aviation Administration, 2012, Integration of Unmanned Aircraft Systems into the National Airspace System - Concept of Operations, Washington D.C., Sep. 28. 
[2] Federal Aviation Administration, 2011, U.S. Department of Transportation, Introduction to TCAS II Version 7.1, Washington D.C., February.

[3] Federal Aviation Administration, 2010, TCAS homepage, http://adsb.tc.faa.gov/TCAS.htm, August.

[4] Federal Aviation Administration, 2000, Introduction to TCAS II Version 7, Washington D.C., November.

[5] Federal Aviation Administration, 2009, Sense and avoid (SAA) for unmanned aircraft systems (UAS), FAA sponsored sense and avoid workshop, October 9.

[6] Consiglio, M., J. Chamberlain, C. Munoz, and K. Hoffler, 2012, Concept of Integration for UAS Operations in the NAS, ICAS, 28th International Congress of the Aeronautical Sciences.

\section{Acknowledgements}

The authors would like to thank Mr. Stephen G. Creaghan, Chief of Aviation Systems Engineering Division (RVT-72) of U.S. DOT Volpe National Transportation Systems Center, for support of this work. The authors also thank Dr. Bruce Wilson and Dr. Seamus McGovern for their technical discussions and comments.

\section{Disclaimer}

The views and opinions expressed in this article are those of the authors and do not necessarily reflect the official policy or position of any agency of the U.S. government. The contents and results in this article should not be utilized in real-world analytic products as they are based only on very limited and dated open source information. Assumptions made within the analysis are not reflective of the position of any U.S. government entity.

\section{Email Addresses}

Y. Zhang, yan.zhang@dot.gov

G. Fairley, gerard.fairley@dot.gov

\section{Appendices}

\section{A. Aircraft Flight Model}

The kinematic algorithm is adopted for the aircraft flight simulation where the horizontal acceleration is created by banking and the vertical acceleration for climb/descent is considered as a constant.

Given an angle of bank ( $\psi)$, the centripetal acceleration $\left(a_{c}\right)$ is given by

$$
a_{c}=\frac{T A S^{2}}{g \tan \psi}
$$

where $g$ is the gravitational acceleration, assuming that the aircraft maintains constant altitude during the banking. On the horizontal plane E-N in the EastNorth-Up coordinate system), given the true heading $(\theta)$, the components of the centripetal acceleration on the East and North axis are, respectively,

$$
\begin{aligned}
& a_{E}=a_{c} \sin \theta+a_{c} \cos \theta \\
& a_{N}=a_{c} \cos \theta-a_{c} \sin \theta
\end{aligned}
$$

Given the acceleration above, the velocity and position of the aircraft can be updated as below.

\section{Velocity Components on ENU Coordinates}

$$
\begin{aligned}
& v_{E}^{i}=T A S_{i-1} \sin \theta+a_{E} d t \\
& v_{N}^{i}=T A S_{i-1} \cos \theta+a_{N} d t \\
& v_{U}^{i}-v_{U}^{i-1}+a_{U} d t
\end{aligned}
$$

\section{Heading Angle}

$$
\theta^{i}=\tan \left(\frac{v_{E}^{i}}{v_{N}^{i}}\right)
$$

\section{Displacement of ENU Coordinates}

$$
\begin{aligned}
& d E=v_{E}^{i} \sin \theta_{i} d t+\frac{1}{2} a_{E} d t^{2} \\
& d N=v_{N}^{i} \cos \theta_{i} d t+\frac{1}{2} a_{N} d t^{2} \\
& d U=v_{U}^{i} d t+\frac{1}{2} a_{U} d t^{2}
\end{aligned}
$$




\section{Displacement of Geodetic Coordinates}

$$
\begin{aligned}
& d \lambda=\frac{d E}{\left(a\left(1-e^{2} \sin ^{2} \phi\right)^{-1 / 2}+h\right) \cos \phi} \\
& d \phi=\frac{d N}{a\left(1-e^{2}\right)\left(1-e^{2} \sin ^{2} \phi\right)^{-3 / 2}+h} \\
& d h=d U
\end{aligned}
$$

\section{Update the Geodetic Coordinates}

$$
\begin{aligned}
& \phi_{i}=\phi_{i-1}+d \phi \\
& \lambda_{i}=\lambda_{i-1}+d \lambda \\
& h_{i}=h_{i-1}+d h
\end{aligned}
$$

\section{B. Indicated Airspeed (IAS) to True Airspeed (TAS) Convert}

$$
T A S=I A S \sqrt{\frac{\rho_{0}}{\rho}}
$$

where $\rho_{0}=\frac{p_{0} M}{R T_{0}}$ and $\rho=\frac{p M}{R T}$ are air pressure at the mean sea level (MSL) and aircraft altitude, respectively. The temperature and the atmospheric pressure at altitude, $h$ in meters, are given by, respectively,

$$
\begin{aligned}
& T=T_{0}-L h \\
& p=p_{0}\left(1-\frac{L h}{T_{0}}\right)^{\frac{g M}{R L}}
\end{aligned}
$$

with parameters defined as

Standard atmospheric pressure at MSL,

$p_{0}=101.325 \mathrm{kPa}$

Temperature lapse rate, $L=0.0065 \mathrm{~K} / \mathrm{m}$

Standard temperature at MSL, $T_{0}=298.15 \mathrm{~K}$

Molar mass of dry air, $M=0.0289644 \mathrm{~kg} / \mathrm{mo}$

Universal gas constant, $R=8.31447 \mathrm{~J} /(\mathrm{mo} \mathrm{K})$

Earth -Surface Gravitational acceleration

$$
g=9.80665 \mathrm{~m} / \mathrm{s}^{2}
$$

\section{Distance between Aircraft}

The line-of-sight (LOS) distance is calculated in the simulation and is given by

$$
d=\sqrt{\left(X_{1}-X_{2}\right)^{2}+\left(Y_{1}-Y_{2}\right)^{2}+\left(Z_{1}-Z_{2}\right)^{2}}
$$

where $\left(X_{i}, Y_{i}, Z_{i}\right)$ is the coordinates of $i$-th aircraft in the Earth-Centered Earth-Fixed coordinate system converted from the geodetic coordinates as below,

$$
\begin{aligned}
& X=\left(\frac{a}{\chi}+h\right) \cos \phi \cos \lambda \\
& Y=\left(\frac{a}{\chi}+h\right) \cos \phi \sin \lambda \\
& Z=\left(\frac{a\left(1-e^{2}\right)}{\chi}+h\right) \sin \phi
\end{aligned}
$$

where $\phi, \lambda$, and $h$ are latitude, longitude, and altitude, respectively; $\chi=\sqrt{1-e^{2} \sin ^{2} \phi}$; and other parameters from WGS-84 elliptical Earth model are listed in Table 4.

Table 4. Parameters for WGS-84 Model

\begin{tabular}{|c|c|}
\hline Parameter & Value \\
\hline Semi-major axis $(a)$ & $6378137.0[\mathrm{~m}]$ \\
\hline $\begin{array}{c}\text { First eccentricity } \\
\text { squared }\left(e^{2}\right)\end{array}$ & $6.69437999014 \times 10^{-3}$ \\
\hline
\end{tabular}

\section{Closure Velocity}

The closure velocity $\left(v_{c}\right)$, is calculated by the difference formula numerically,

$$
v_{c}=\frac{d_{i}-d_{i-1}}{d t}
$$

where $d_{i}$ is the LOS distance between aircraft, and $d t$ is the time duration between time step $i$ and $i-1$. When $v_{c}>0$, it indicates that the distance between the two aircraft increases; while $v_{c}<0$ means that the distance between the two aircraft decreases.

\section{Integrated Communications Navigation and Surveillance (ICNS) Conference}

April 23-25, 2013 